\title{
Torsion of a Wandering Spleen Presenting as a Painful Pelvic Mass Post Pregnancy: Imaging Diagnosis
}

\author{
Abbey $\mathbf{P}^{1}$, Aarushi $\mathbf{A}^{1}$, Andley $\mathbf{M}^{2}$, Anand $\mathbf{R}^{1}$ \\ ${ }^{1}$ Department of Radio-Diagnosis, ${ }^{2}$ Department of Surgery, Lady Hardinge Medical College \\ and associated hospitals, New Delhi - 110001, India
}

\begin{abstract}
A 'wandering' spleen is an uncommon condition most often found in young women, and may be incidentally detected or present acutely with splenic torsion. We report the case of a young woman who noticed an abnormal suprapubic lump after her first pregnancy, and subsequently presented seven months later with acute pain and sudden increase in size of the mass due to torsion of a pelvic spleen. We highlight the typical ultrasound and CT (computed tomography) imaging findings which helped to make a confident pre-operative diagnosis. Splenectomy was performed and confirmed the development of splenic infarction.
\end{abstract}

Key words: Wandering Spleen, Splenic torsion, Splenic infarct, CT whirl sign

\section{Introduction}

A 'wandering' spleen is an uncommon condition in which hypermobility of the spleen results from laxity of its peritoneal attachments, and the splenic vascular pedicle gets elongated. These cases are at risk of splenic torsion, which is a surgical emergency as it can progress to infarction.

Few case reports in the literature describe the presentation of these patients with acute abdomen during pregnancy. ${ }^{1,2}$ We describe the case of a young woman who developed an intermittently painful suprapubic mass

Correspondence to: Pooja Abbey, M.D., Assistant Professor, Department of RadioDiagnosis, Lady Hardinge Medical College and associated hospitals, New Delhi 110001, India

E mail Id: pooja_abbey@yahoo.co.in which she noted post delivery of her first child, and subsequently presented with an acute abdomen seven months later, due to splenic torsion affecting an ectopic spleen. Accurate diagnosis was possible based on ultrasound and computed tomography (CT) findings.

\section{Case Report}

A twenty four years old lady presented to the surgical emergency with a painful suprapubic mass. She had given birth to a healthy full term baby (her first) by a normal vaginal delivery seven months before. Subsequently, she noticed abnormal fullness in the suprapubic region which persisted and was accompanied by intermittent episodes of pain. Ten days prior to presentation, the pain had become constant and increased in severity, along with increase in the size of the mass. She also had a past history of few 


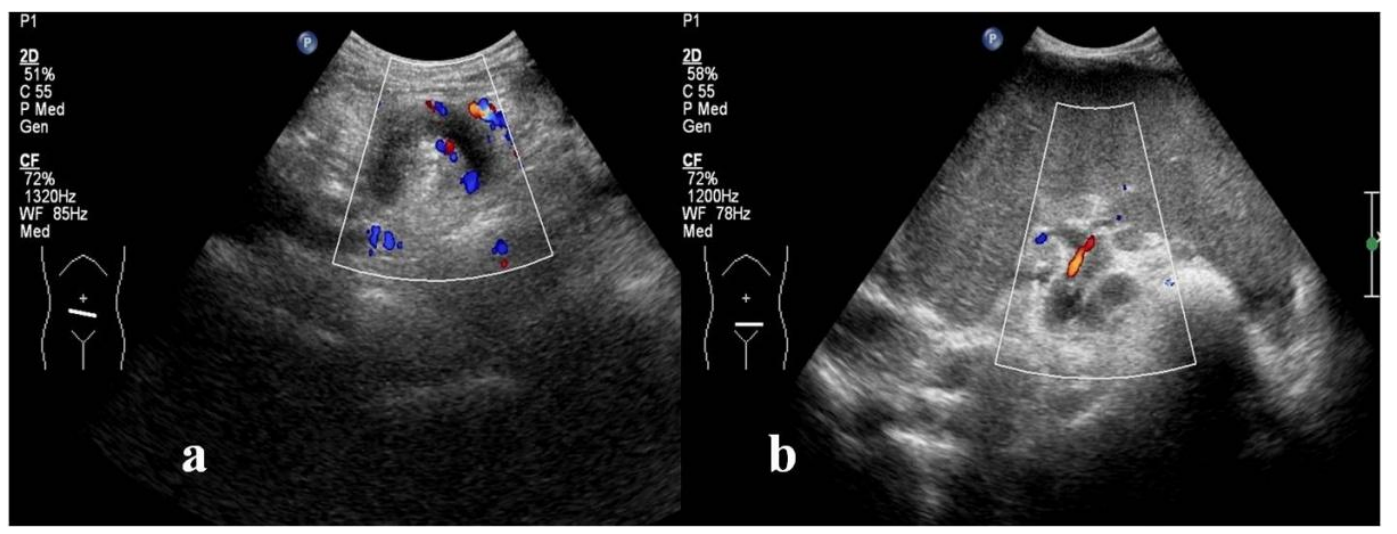

Fig. 1: Ultrasound with colour Doppler shows twisting of the splenic vascular pedicle and a dilated splenic vein with no colour flow within (a). The spleen is visualized in the pelvis and arterial flow is seen at the splenic hilum (b).

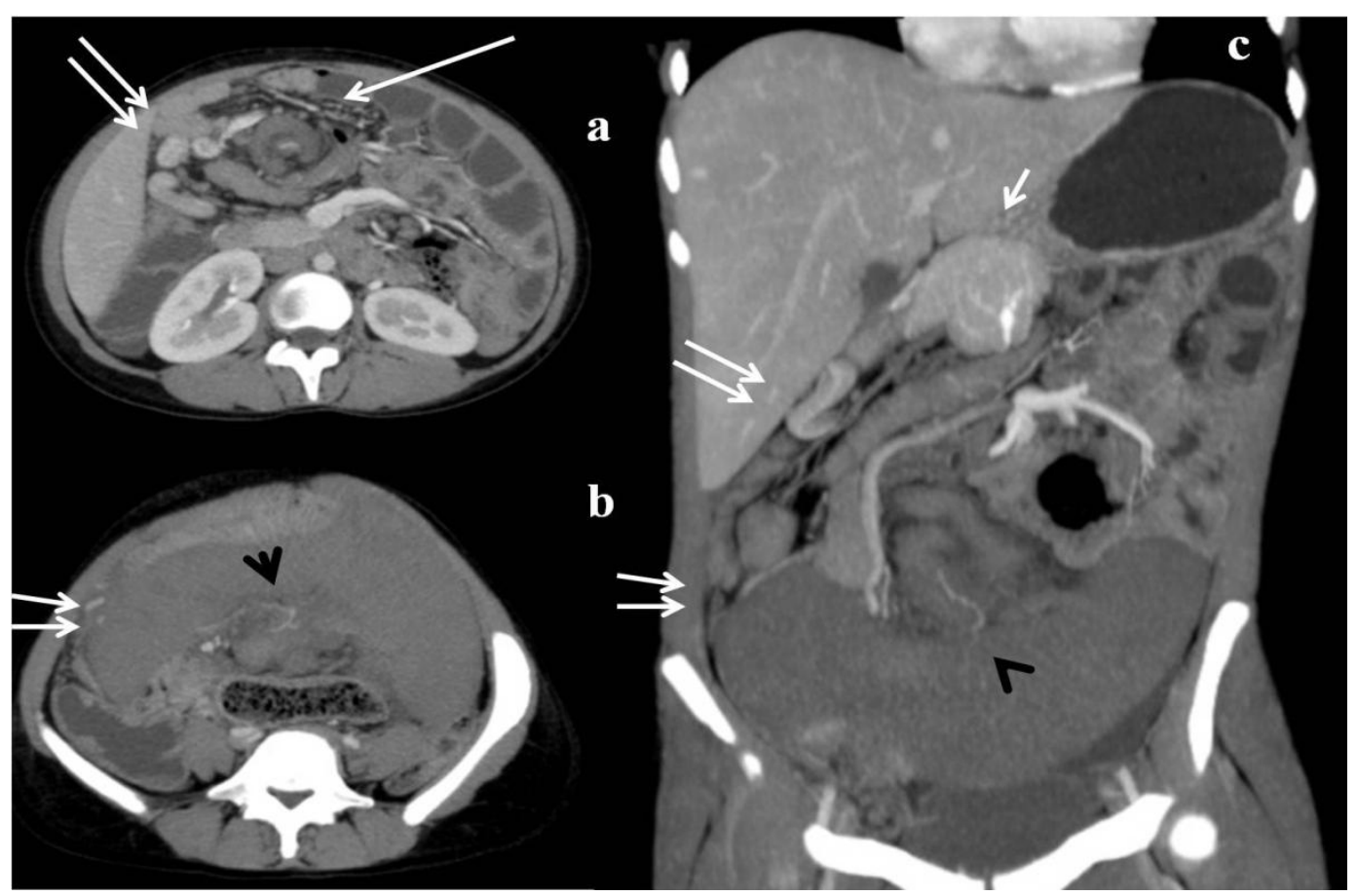

Fig. 2: CECT images. Axial image (a) shows the CT 'whirl' sign (long arrow). The twisted pedicle shows no enhancement of the dilated splenic vein. Spleen shows minimal peripheral enhancement $(b, c)$ with opacification of splenic artery at its hilum (black arrowheads). The tail of pancreas is intraperitoneal (short arrow in c). Multiple venous collaterals are seen in the perisplenic region and right upper abdomen (double arrows).

episodes of colicky pain abdomen, which had resolved spontaneously and had not been investigated.

At presentation, she was found to have a tender lump in the hypogastric region. She was pale, afebrile and her blood pressure was normal. Her urine pregnancy test was negative. Laboratory investigations revealed an elevated total leucocyte count.

An abdominal ultrasound revealed absence of spleen in the left upper quadrant. An enlarged heteroechoic spleen was visualized in the pelvis, lying just cranial to the urinary bladder (Fig. 1). A twisted tubular structure 
was seen in the central abdomen, which was traceable till the hilum of the ectopic spleen. It showed presence of arterial flow on Doppler imaging; however, no venous flow was detectable (Fig. 1).

A contrast enhanced CT (computed tomography) scan was performed and confirmed the ectopic location of the spleen, which was enlarged and showed only minimal heterogeneous contrast enhancement of its peripheral parenchymal and subcapsular regions (Fig. 2). The splenic vein was dilated and showed complete lack of contrast enhancement. Multiple twists of the splenic vascular pedicle were present, giving a 'whorled' appearance on CT. Multiple venous collaterals were seen in the perisplenic region. The pancreatic tail was intraperitoneal in location.

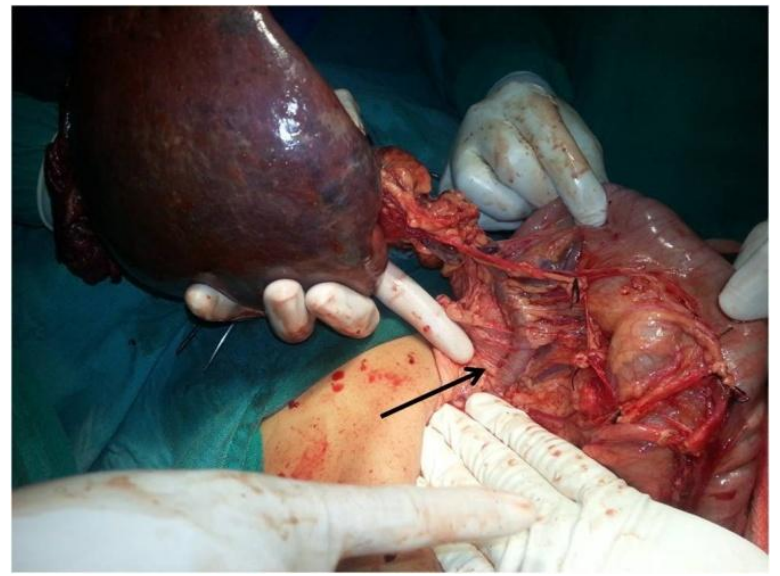

Fig. 3: Peroperative photograph shows the enlarged, infracted spleen. The splenic vein is dilated and thrombosed (black arrow).

Imaging features were diagnostic of splenic torsion. The patient underwent a laparotomy, which revealed an enlarged, infarcted pelvic spleen. Its vascular pedicle was twisted and the splenic vein was dilated and thrombosed (Fig. 3). A splenectomy was performed and histopathology confirmed infarction.

\section{Discussion}

A 'wandering' spleen is an uncommon entity in which the spleen is found in an ectopic position within the abdominopelvic cavity. Acquired laxity or congenitally abnormal development of supporting ligaments makes the spleen excessively mobile and causes it to 'wander'.

This condition may sometimes present in childhood, affecting boys more often than girls. ${ }^{3}$ More commonly, it is diagnosed in young adults, and adult women are affected more than men. Hormonal influences are thought to play a role in the development of acquired ligamentous laxity, with increased predisposition in multiparous or peripartum females. Isolated case reports describe the condition presenting as an acute abdomen during pregnancy. ${ }^{1,2}$

The clinical presentation is extremely variable, and ranges from an asymptomatic palpable mass, to intermittent attacks of pain abdomen, to an acute abdomen. ${ }^{4}$ The abnormally long splenic vascular pedicle is at risk for getting twisted, which may result in splenic torsion.

Torsion may be incomplete or complete, transient/ intermittent or constant. The clinical symptomatology depends on the presence and degree of torsion. Intermittent pain abdomen can occur as a result of repeated episodes of torsion followed by spontaneous de-torsion. Persistent torsion of the pedicle initially causes obstruction of the low pressure flow in the lymphatics, followed by the venous outflow. ${ }^{5}$ This results in edema and can result in venous infarction. Splenic congestion and enlargement causes stretching of the capsule, and results in pain. 
There is preservation of high pressure arterial flow to begin with, but long standing high grade torsion can cause compromise of the arterial inflow as well.

Imaging studies play a vital role in the diagnosis of this condition, and its associated complications. $^{6-9}$ The diagnosis may be suspected on ultrasound scans by noting absence of spleen from its usual expected location in the left upper quadrant. The ectopic spleen may show a normal ultrasound appearance, or may be enlarged and heteroechoic in appearance. The demonstration of a twisted vascular pedicle, while specific for splenic torsion, may be difficult on ultrasound scans due to abdominal tenderness and obscuration by bowel gases. Doppler imaging may be helpful in documentation of presence or absence of arterial and venous flow within the splenic vascular pedicle.

CT is a valuable imaging tool in the diagnosis of a wandering spleen, as well as splenic torsion. CT can demonstrate the exact location of the ectopic spleen and delineate its vascular supply. A valuable evidence of a hypermobile/ wandering spleen on CT is the demonstration of an intraperitoneal location of the tail of pancreas. This sign is helpful in making the diagnosis in cases which present as an incidental CT finding. Occasionally, cases may be complicated by associated pancreatitis or pancreatic necrosis. ${ }^{3}$ Patients with splenic torsion demonstrate enlargement of the spleen, and poor/ absent enhancement of the parenchyma is seen if there is associated splenic infarction. Thrombosed splenic vessels may appear hyperdense on non contrast CT. Perisplenic venous collaterals can also be detected on
$\mathrm{CT}$ in cases with chronic torsion, as were seen in our case.

The CT 'whirl' sign has been described as a specific finding seen in cases with torsion. ${ }^{8}$ This sign refers to the whorled appearance of the twisted splenic vascular pedicle with concentric bands of higher and lower CT density. The splenic vessels form the innermost structures within the torsed pedicle, and are surrounded by peritoneal folds and fat.

A confident imaging diagnosis of splenic torsion complicating a wandering spleen can be made in cases with typical CT findings, as in our case. Splenic torsion is a surgical emergency, as it can lead to infarction if not promptly treated.

Definite management of a wandering spleen is surgical, either by splenopexy or splenectomy. The type of surgery depends on the clinical presentation, presence of torsion and the intra-operative assessment of splenic viability after de-torsion. In cases with an acute presentation or a non-viable spleen, splenectomy is the procedure of choice and may be performed via a laparotomy or a laparoscopic approach. ${ }^{10}$

\section{References}

1. Parvaiz A, Chandran S, Karim A, Kumar K, Jeffrey P, Lagattolla NRF. Torted and ruptured wandering spleen presenting as a surgical emergency in pregnancy. The Scientific World Journal 2004;4:1035-7.

2. Gilman RS, Thomas RL. Wandering spleen presenting as acute pancreatitis in pregnancy. Obstet. Gynecol 2003;101: 1100-2. 
3. Brown CVR, Virgilio GR, Vazquez WD. Wandering spleen and its complications in children: a case series and review of the literature. J Pediatr Surg 2003;38: 1676-9.

4. Buehner M, Baker MS. The wandering spleen. Surg Gynecol Obstet 1992;175: 373-87.

5. Lubner MG, Simard ML, Peterson CM, Bhalla S, Pickhardt PJ, Menias CO. Emergent and nonemergent nonbowel torsion: Spectrum of imaging and clinical findings. Radiographics 2013;33:155173.

6. Swischuk LE, Williams JB, John SD. Torsion of wandering spleen: the whorled appearance of the splenic pedicle on CT. Pediatr Radiol 1993;23: 476-477.
7. Nemcek AA, Miller FH, Fitzerald SW. Acute torsion of a wandering spleen: diagnosis by CT and duplex Doppler and color flow sonography. Am J Roentgenol 1991;157:307-9.

8. Raisssaki M, Prassopoulos P, Daskalogiannaki M, Magkanas E, Gourtsoyiannis N. Acute abdomen due to torsion of wandering spleen: CT diagnosis. Eur Radiol 1998;8:1409-12.

9. Ben Ely A, Zissin R, Copel L et al. The wandering spleen: CT findings and possible pitfalls in diagnosis. Clin Radiol 2006;61:954-8.

10. Benevento A, Boni L, Dionigi G, Ferrari A, Dionigi R. Emergency laparoscopic splenectomy for 'wandering' (pelvic) spleen: case report and review of the literature on laparoscopic approach to splenic disease. Surg. Endosc 2002;16:1364-5. 